\title{
Research on the causes and Countermeasures of the tension between doctors and patients
}

\author{
Xi Chen \\ School of Management, Wuhan University of Technology, Wuhan 430000, China. \\ 113552969@qq.com
}

Keywords: Doctor-patient relationship, Conflict between doctors and patients, Medical reform, Crisis management.

\begin{abstract}
In recent years, with the continuous development of China's economy and the improvement of people's living standard, people's attention to health has been strengthened. But because of our limited medical resources, uneven distribution of medical resources, uneven level of doctors and other reasons, led to the difficult and expensive problem, also led to the doctor's working strength is too large, the job satisfaction is not high problems have become increasingly prominent. In this case, the doctor-patient relationship has become an unprecedented tension, the doctor-patient conflicts have occurred, resulting in a huge adverse impact. This paper combines the research scholars at home and abroad, expounds the connotation and current situation of the doctor-patient relationship, analyzes the reason for tense doctor-patient relationship, and put forward the development strategy from the short-term and long-term perspective, to provide ideas for easing the doctor-patient relationship.
\end{abstract}

\section{Introduction}

Doctor-patient relationship has always been an important interpersonal relationship. In ancient China and the west, there are many simple discussions on how to deal with the relationship between doctors and patients. Since the implementation of reform and open policy in China, has undergone profound changes in the doctor-patient relationship, the improvement of living standards must be accompanied by the health needs of the deepening, however, in the past more than and 20 years, the doctor-patient relationship has emerged the tense situation of hitherto unknown. On the one hand, with the development of economy, at the expense of the environment effect gradually, people's health needs gradually increased, but because of the existing medical infrastructure and doctors and conditions, medical treatment is difficult and expensive, such as the poor doctor experience gradually formed and prominent, caused the dissatisfaction of the masses; On the other hand, because of China's health care system is not perfect and lack of medical resources and other practical reasons, which leads to the problem of doctors work pressure, poor working environment, investment and income does not match, the doctor group generally feel job satisfaction is not high. In this case, the doctorpatient relationship becomes more severe, and there is a serious problem of violent conflicts between doctors and patients. The world's authoritative medical journal "Lancet" published entitled "Chinese doctors are under threat" article, elaborated the Chinese doctors often become victims of amazing building violence in their own work, caused great repercussions and attention at home and abroad ${ }^{[1]}$. Many events are frequent conflicts between doctors and patients occupy the headlines, often lead to public discussion, and these conflicts behind, is often injured patients and doctors, doctor-patient relationship has become the focus of academic research and the focus of attention of the whole society. Based on the research of domestic and foreign scholars, this paper analyzes the causes of tension between doctors and patients from the perspective of internal and external, and puts forward the countermeasures to improve the doctor-patient relationship. 


\section{The connotation and development of doctor-patient relationship}

\subsection{Connotation of doctor-patient relationship}

Doctor-patient relationship is one of the most important interpersonal relationships. Health needs are the eternal needs of mankind, so that medical practice has become one of the most important practical activities of human beings. The doctor-patient relationship has two kinds of understanding of the narrow and broad sense: the doctor-patient relationship refers to doctors and patients individual relationship; generalized doctor-patient relationship refers to the medical staff as the center of the medical service and include all relevant parties, and as the center of the multiple relationship between group and group of a party there is a direct relationship between the health and interests of all patients including patients ${ }^{[2]}$.The doctor-patient relationship is divided into two parts: technical and nontechnical. Non technical relationship refers to the relationship between social and psychological aspects of medical personnel and the treatment process, in the process of medical treatment of medical effect has invisible effect.

Szasz and Hollender from the perspective of medical psychology, the relationship between doctors and patients can be divided into active and passive type, Guidance and cooperation type and Joint participation type ${ }^{[3]}$.(1) active and passive. The doctor was fully active, authoritative and completely passive physicians without any doubt, the patient does not raise any objection.(2) Guidance and cooperation. Doctors and patients have the initiative, the physician's opinion was respected, but the patient may have doubts and seek to explain.(3) Joint participation type.The initiative of doctors and patients with common participation in decision-making and implementation of health. The physician's views are often involved and living habits, and interpersonal adjustment, patient cooperation and self treatment is particularly important.

Doctors and patients are independent individuals. In the course of the treatment, the two sides are prone to disagreement, friction and even conflict, the definition of the relationship between doctors and patients is to understand the causes of tension between doctors and patients and put forward the premise of effective countermeasures.

\subsection{Current situation of doctor-patient relationship in China}

Since the reform and opening up to the outside world, the research on the relationship between doctors and patients in our country has gone through 3 stages:

(1) From 1978 to early 1990s. This stage mainly discusses the relationship between doctors and patients from the hospital, the focus of the study was to increase the hospital construction, strengthen medical ethics education.(2) Early 1990s to early twenty-first Century. This stage mainly from the two aspects of the hospital and patients to explore the issue of doctors and patients, the focus of the study is to strengthen the construction of the hospital and the patient's rights to protect themselves.(3) Early twenty-first Century to the present. This stage is mainly to interpret the doctor-patient relationship from multiple levels of doctor and patient, medical system and society, and make relevant reflection on the marketization reform of the hospital.

At the present stage, the relationship between doctors and patients is becoming more and more serious, there is a crisis of trust between doctors and patients. Nationally, the number of medical disputes has increased significantly, growing at an annual rate of about $30 \%$, but not proportional to medical malpractice ${ }^{[4]}$

\section{Cause of tension between doctors and patients}

\subsection{Internal causes}

(1)Hospital factor. (1)Self-protection consciousness. In the course of diagnosis and treatment, the majority of doctors are self-protection awareness, the first consideration is to ensure the safety of medical care, to ensure that the medical risk is reduced to the minimum ${ }^{[5]}$. Doctors in order to minimize the risk of medical treatment, to take over inspection and excessive medical behavior, resulting in increased economic burden of patients, the doctor-patient relationship deteriorated.(2) Lack of professional ethics of doctors. The current medical education system mainly focuses on the medical skills education, the lack of humanistic education, resulting in some medical personnel 
quality is low, lack of training. In addition, part of the medical staff of patients with a lack of patience and compassion, sense of responsibility is not strong, poor service attitude. (3) Limited technical level. Some medical personnel technical level is not high, the lack of clinical experience, often Misdiagnosis; application of some drug indications, methods, contraindications and incompatibility are not familiar with, resulting in serious consequences and mistreatment of medical disputes.

(2) Patient factors. (1)Enhance awareness of rights. With the popularization of the law in our country, people's awareness of law and protection of rights has been strengthened. If patients and their families in the process of medical treatment of hospital or medical staff of medical service quality, service attitude and so on, they will use the law to safeguard their rights and interests, leading to the increase of medical disputes. (2)Expect too much medical effect but lack of medical knowledge.

Although the rapid development of modern medicine, but also has its particularity and limitations of the medical treatment of some diseases is not ideal. However, some patients and their families for the particularity of medicine of lack of knowledge, lack of medical knowledge, the medical effect of high expectations, as long as in the hospital after treatment, auxiliary examination and the doctor a series of scientific instruments, we will be able to cure the disease, but this is beyond the scope of today's medical field to reach. When they pay high medical costs and reach the desired therapeutic effect, it will vent their anger in the hospital and the doctor concerned, causing tension between doctors and patients.

(3) Doctor-patient relationship factor. There are some problems such as information asymmetry, lack of trust and poor communication between doctors and patients, these factors will make the doctor-patient relationship becomes tense.

\subsection{External cause}

(1) Defects of medical system in china. First of all, China's health care is a social undertaking, however, with the transformation of economic system, public hospitals market gradually, government financial investment is reduced, appeared to rely mainly on the treatment fee to maintain the operation and development of the masses of ${ }^{[6]}$. Secondly, the legal system construction of the doctor-patient relationship in our country is lagging behind, and it is often difficult to follow the rules of medical disputes. This makes the doctor in order to reduce the risk of medical treatment, to avoid medical disputes, patients have to be more open to improve the diagnostic rate, which is an important reason for the increasing number of patients diagnosed with the project. Finally, the public opinion and news reports tend to vulnerable groups, in the medical dispute in general, regardless of whether the source of fault in medical patients is often the attention of the party, the spearhead of public opinion often point to doctors, which also caused a public distrust of doctors, the doctor-patient relationship is more severe.

\section{Development countermeasure}

\subsection{Short-term Countermeasures.}

In the short term, China's strained doctor-patient relationship is difficult to thoroughly improve the doctor-patient conflicts have occurred, has brought great harm to the doctors, patients and the public. The characteristics of the conflict between doctors and patients are in line with the characteristics of crisis management in crisis management, so we can explore and deal with it from the perspective of crisis management. Based on the concept of crisis management, we can establish the Doctor-patient Conflict control system, the Doctor-patient Conflict is divided into three stages of conflict prevention, conflict management, conflict aftermath to control ${ }^{[7]}$, as shown in fig 1. 


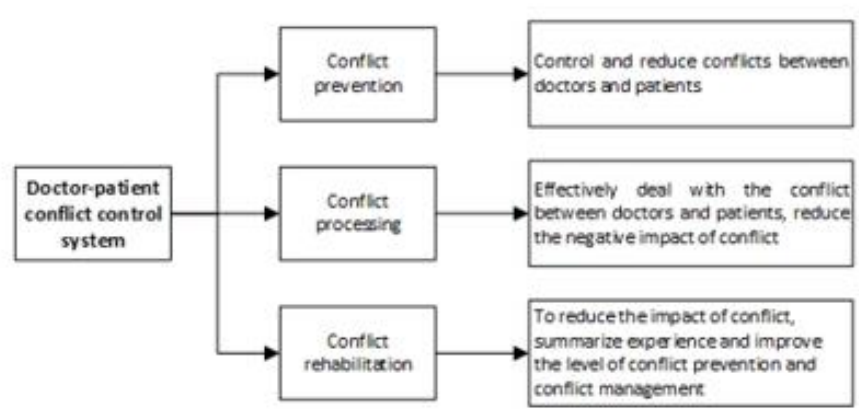

Fig. 1 The doctor-patient conflict control system based on Crisis management

(1)Conflict prevention phase. The purpose of this stage is to use a series of management measures to control and reduce the occurrence of conflicts between doctors and patients. Establish and improve the Doctor-patient Conflict Prevention and control network, the operation of modern big data and monitoring technology, strengthen the ability to deal with the information, the establishment of the Doctor-patient Conflict resolution plan, improve the early warning system and mechanism. Analysis and study of the status quo of doctor-patient relationship, to capture the tension between doctors and patients, the situation may lead to conflict, early intervention, thereby reducing the escalation of conflict between doctors and patients, to reduce the risk of conflict.

(2) Conflict processing phase. The improper handling of the conflict between doctors and patients will bring huge losses to the society, the public, the government and the medical and health service system. Encountered sudden clashes between doctors and patients, should be in accordance with the emergency plan for fast, by psychological experts, security personnel, police, lawyers, government officials such as the composition of the ad hoc group of specialized and full range of conflict processing, to avoid the escalation of the conflict, to reduce the negative impact of conflict as much as possible.

(3) Conflict rehabilitation phase. First of all, the Doctor-patient Conflict Resolution, because the media and public opinion and other factors, the negative social impact will still exist, so we should strengthen the conflict management transparency, avoid public opinion distortion, have a negative impact; secondly, to deal with the conflict between doctors and patients to conduct a comprehensive analysis, summarize the experience and lessons, to improve the level of conflict prevention and treatment.

\subsection{Long-term strategy}

If we want to fundamentally change the tension between doctors and patients, we must carry out long-term and in-depth health care reform, take the government as the leading role, and the doctors and patients to participate together, so as to achieve the fundamental goal of reform.(1) The hospital should change the current mode of diagnosis and treatment, gradually changed from the original "disease centered" model of diagnosis and treatment for the "patient-centered" model of diagnosis and treatment of patients, more humanistic care, enhance service awareness, improve the overall quality of medical care. At the same time, the medical staff to improve the level of business, to understand the latest academic trends, grasp the forefront of medical knowledge and skills, learning advanced medical technology. And the hospital should establish incentive system, improve the working environment of medical staff, at the same time, establish the service evaluation system, investigate the patients' satisfaction, strengthen the communication between doctors and patients.(2) Patients on the one hand need to learn medical and legal knowledge, so that patients and their families understand the risks of medical treatment, reduce information asymmetry, strengthen legal advocacy, improve the legal consciousness, to solve the problems encountered by legal means, to avoid contradictions; on the other hand, should also strengthen the patient's self-cultivation, strengthening the medical personnel in respect of ${ }^{[8]}$. (3) The government should increase investment in medical institutions, so that medical institutions return to public welfare. And it should strengthen the functions of the effective implementation of supervision, reduce the public medical institutions commercial and economic interests of the development trend, ensure the public medical institutions, social welfare and welfare nature and role, so that the medical institutions to realize the true meaning 
of the public. It is necessary to make the distribution of medical and health resources balanced, strengthen macro-control, and achieve the optimal allocation of regional health resources ${ }^{[9]}$. The government needs to continue to improve the health care system, to reduce the public's economic burden of medical care, but also to improve the relevant laws and regulations related to medical disputes. As soon as possible to develop and implement the medical service law, the law on the protection of patients rights and interests, through legislation to clear the limits of the rights of both doctors and patients.

\section{Summary}

The doctor-patient relationship is one of the most important interpersonal relationships, which has a great influence on social stability and development. In recent years, the doctor-patient relationship is becoming more and more intense in our country, the doctors are not satisfied with the working environment, and the conflicts often erupt between doctors and patients. The main reason for the tension between doctors and patients comes from two aspects: internal and external: The doctorpatient relationship from the internal point of view, both doctors and patients as the main body of the doctor-patient relationship, any problems may lead to the disharmony, such as medical skill, occupation morality is not enough, cause patient's lack of common sense and trust etc; From the perspective of doctor-patient relationship, the defects of the medical system, the imperfect relevant laws and the unclear orientation of public opinion make the doctor-patient relationship increasingly tense.

To improve the doctor-patient relationship, we need to focus on two aspects of the short and long term. In the short term, we should establish the Doctor-patient Conflict Control System from conflict prevention, conflict management, conflict with the three aspects to deal with the conflicts between doctors and patients, to reduce the negative impact of the conflict and reduce conflict; In the long term, we should deepen the medical reform, transformation of hospital medical treatment mode, improve the professional level, improve the humanistic care for patients, strengthen the popularization of medical knowledge and legal concepts, improve the medical resources, optimize the allocation of medical resources, improve the relevant laws. So as to fundamentally improve the doctor-patient relationship, to maximize the interests of both doctors and patients.

\section{References}

[1]. Lancet T. Chinese doctors are under threat [J]. Lancet, 2010, 376(9742):657.

[2]. B.Huang. "Research on the relationship between doctors and patients in the perspective of new medical reform," Guangxi Medical University, China, 2010.

[3]. Szasz T, Hollender M. The basic models of the doctor-patient relationship[J]. 1956.

[4]. W. J. Zhang, Y. H. Hao and Q. H. Wu. Medicine and Society, 2014, 27 (4) :44-46.

[5]. X.X. Han. Gansu Science and Technology, 2010, 26(12):169-172.

[6]. J.J. iao, D. Huang. Journal of Traditional Chinese Medicine Management. 2008, 16(9):658-660.

[7]. L.J .Li. Medicine and Philosphy. 2013, 34(15):70-73.

[8]. W. J. Zhang, Y. H. Hao, Q. H. Wu. Medicine and Society, 2014, 27(4):44-46.

[9]. J.H. Fan. China Management Informationization, 2013, 16(5):71-71. 